\title{
„Ich fühle mich extrem wohl damit, wenn das was wir vermarkten, gut erforscht ist"
}

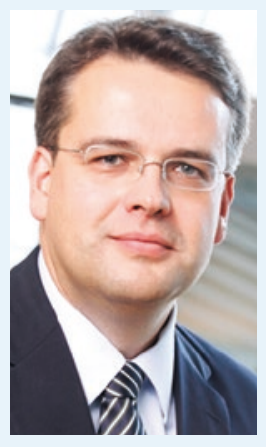

G. Mastio

\begin{abstract}
Gérald Mastio ist seit Januar 2014 Geschäftsführer der CP GABA GmbH, bereits seit August $\mathbf{2 0 1 2}$ leitete er das Vorgängerunternehmen GABA GmbH. Im Interview mit der ZWR gibt der gebürtige Elsässer Einblicke in seine Arbeit als Geschäftsführer, die Alleinstellungsmerkmale von CP GABA und wie er seine Balance behält.
\end{abstract}

\begin{abstract}
? Herr Mastio, Sie sind seit Januar 2014 Geschäftsführer der CP GABA $\mathrm{GmbH}$, was macht Ihnen besonders Spaß an Ihrer Arbeit?

Mastio: Die Arbeit mit den Kollegen. Wir haben ein tolles Team hier in Deutschland. Jeder meiner Mitarbeiter hat seinen Anteil daran, dass CP GABA heute so gut in Deutschland da steht.
\end{abstract}

? Was ist für Sie die größte Herausforderung als Geschäftsführer?

Mastio: Nach der organisationalen Zusammenführung von Colgate-Palmolive und GABA stehen wir vor der Herausforderung, aus 2 Unternehmenskulturen eine zu machen. Glücklicherweise gibt uns die Konzernführung die Möglichkeit, unsere Strukturen den lokalen Gegebenheiten anzupassen. Als Geschäftsführer fühle ich mich verantwortlich, einen Rahmen zu geben, damit sich sowohl die Mitarbeiter als auch das Unternehmen stetig und gesund weiterentwickeln können.

\section{? Welche unternehmerischen}

Schwerpunkte setzen Sie in Ihrer Arbeit bei CP GABA?

Mastio: Da sind zunächst die klassischen unternehmerischen Aufgaben eines Geschäftsführers, wie beispielsweise daran zu arbeiten, unternehmerische Prozesse schlanker und einfacher zu machen. Wichtig ist, dass sich alle auf die wesentlichen Prioritäten konzentrieren können und das Organisatorische oder Formale möglichst wenig Raum einnimmt. Ein weiteres Herzensthema von mir ist aber auch die Verbesserung der internen Feedbackschleifen. Bereits heute führe ich mit jedem meiner Mitarbeiter mindestens 2-mal im Jahr ein 4-Augen-Gespräch, um ungefiltert und direkt einen Eindruck zu bekommen, was jedem wichtig ist.

? Inwiefern können Sie die Erfahrungen aus Ihrer vorherigen Tätigkeit in einem forschenden Pharmaunternehmen bei CP GABA einbringen?

Mastio: Ich profitiere sehr stark von meinen früheren Erfahrungen in der forschenden Pharmaindustrie, denn auch bei CP GABA haben wir einen hohen Qualitätsanspruch an unsere Produkte. Wir entwickeln unsere Produkte für die Mundgesundheit auf einem wissenschaftlichen Fundament und testen ihre Wirkung in validen Studien.

\section{? Welchem Leitgedanken folgt} CP GABA bei der Entwicklung und Herstellung seiner Produkte?

Mastio: Wie bereits gesagt arbeiten wir auf einer wissenschaftlichen Basis und in enger Abstimmung mit der Profession, um die hohe Qualität unserer Mundpflege-Produkte sicherzustellen. Die hervorragende Produktentwicklung von GABA konnte durch die organisationale Zusammenführung mit Colgate Palmolive nochmals verbessert werden. Colgate verfügt über mehrere ausgezeichnete Technologie-Zentren mit über 200 Wissenschaftlern, Klinikern und Ingenieuren weltweit. Ich fühle mich extrem wohl damit, wenn ich weiß, dass das, was wir vermarkten, gut erforscht ist.

? Welche zentralen Vorteile sehen Sie in der engen Zusammenarbeit mit den Fachgesellschaften?

Mastio: Über unsere engen und langjährigen Kooperationen mit den Fachgesellschaften sowie über unseren Außendienst sind wir ganz nah an den Praktikern. Mit Zahnärzten und den Prophylaxe-Teams im Austausch zu sein, ist wichtig, um zu erfahren, was die Profession von uns, der
Industrie, braucht. Dialog ist dabei das Schlüsselwort. Über viele Jahre ist hier ein vertrauensvolles Miteinander entstanden. Wir schätzen diese Zusammenarbeit besonders, weil sie uns dabei hilft, bedarfsorientierte, fundiert erprobte Produkte zu entwickeln, die auch der Prüfung von Experten standhalten.

? Welche Erfolge hat die dentale Prophylaxe vorzuweisen und was kann in den kommenden Jahren erreicht werden?

Mastio: Auch wenn es inzwischen vielleicht einige nicht mehr hören können, aber ein nach wie vor wichtiges Pfund in der Prophylaxe sind die Fluoride. Sie waren und sind auch weiterhin ein grandioser Erfolg. Bei der kontinuierlichen Suche nach weiteren Ausbaumöglichkeiten der Prophylaxe sind wir auf die PRO-ARGIN ${ }^{\circledR}$ Technologie gestoßen. Sie wird kombiniert mit Fluoriden in unserem Zahnpasta-Produkt elmex ${ }^{\circledR}$ Kariesschutz Professional angewendet. Wie gut diese Idee ist, zeigen die überzeugenden Daten der klinischen Studien zu dieser Zahnpasta.

\section{? Herr Mastio, wie sorgen Sie für} Ihre Work-Life-Balance bei dieser anspruchsvollen Arbeit?

Mastio: Am Liebsten bei meiner Familie im Elsass. Meine Frau, meine Kinder und unser Hund sorgen dafür, dass ich den nötigen Ausgleich habe. Wir verbringen viel Zeit gemeinsam und genießen die guten Lebensbedingungen der Region, vor allem das gute Essen. Für meine Tochter, die gerade im Teenager-Alter ist, bin ich hauptsächlich der Party-Chauffeur, aber das ist okay.

Herr Mastio, vielen Dank für das Interview.

Im Auftrag der ZWR führte das Gespräch Erika Hettich

Diese Rubrik ist entstanden mit freundlicher Unterstützung der CP GABA GmbH, Hamburg 